International Journal of Pure and Applied Mathematics

Volume 115 No. $1 \quad 2017,27-41$

ISSN: 1311-8080 (printed version); ISSN: 1314-3395 (on-line version)

url: http://www.ijpam.eu

doi: 10.12732 /ijpam.v115i1.3

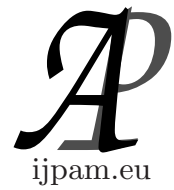

\title{
STRONGLY T-SEMISIMPLE MODULES AND STRONGLY T-SEMISIMPLE RINGS
}

\author{
Inaam Mohammed Ali Hadi ${ }^{1}$, Farhan Dakhil Shyaa ${ }^{2} \S$ \\ ${ }^{1}$ Department of Mathematics \\ University of Baghdad \\ Baghdad, IRAQ \\ and \\ College of Education for Pure Sciences (Ibn-Al-Haitham) \\ University of Baghdad, Baghdad, IRAQ \\ ${ }^{2}$ Department of Mathematics University of Al-Qadisiyah \\ College of Education, Al-Qadisiya, IRAQ
}

\begin{abstract}
In this paper, we introduce the notions of strongly t-semisimple modules and strongly t-semisimple rings as a generalization of semisimple modules, rings respectively. We investigate many characterizations and properties of each of these concepts. An R-module is called strongly t-semisimple if for each submodule $\mathrm{N}$ of $\mathrm{M}$ there exists a fully invariant direct summand $\mathrm{K}$ such that $\mathrm{K}$ t-essential in $\mathrm{N}$. Also, the direct sum of strongly t-semisimple modules and homomorvarphic image of strongly t-semisimple is strongly t-semisimple.

$\mathrm{A}$ ring $\mathrm{R}$ is called right strongly t-semisimple if $\mathrm{R}_{R}$ is strongly t-semisimple. Various characterizations of right strongly t-semisimple rings are given.
\end{abstract}

AMS Subject Classification: 16D10, 16D70, 16D90, 16P70

Key Words: strongly t-semisimple, t-semismiple modules

\section{Introduction}

Through this paper $R$ be a ring with unity and $M$ is a right R-module. Let

Received: December 25, 2016

Revised: $\quad$ March 22, 2017

Published: June 24, 2017

(c) 2017 Academic Publications, Ltd. url: www.acadpubl.eu

$\S_{\text {Correspondence author }}$ 
$Z_{2}$ (M) be the second singular (or Goldi torsion) of $M$ which is defined by $Z(M /(Z(M)))=\left(Z_{2}(M)\right) /(Z(M))$ where $Z(M)$ is the singular submodule of $M A$ module $M$ is called $Z_{2}$-torsion if $Z_{2}(M)=M$ and a ring $R$ is called right $Z_{2}$-torsion if $Z_{2}\left(R_{R}\right)=R_{R}[8]$., A submodule $A$ of an $R$-module $M$ is said to be essential in $M$ (denoted by $A \leq_{e s s} M$ ), if $A \bigcap W(0)$ for every nonzero submodule $W$ of $M$. Equivalently $A \leq_{\text {ess }} M$ if whenever $A \bigcap W=0$, then $W=0$ [9],Asgari and Haghany [4] introduced the concept of $t$-essential submodules as generalization of essential submodules. A submodule $N$ of $M$ is said to be $t$-essential in $M$ (denoted by $\left(N \leq_{\text {tes }} M\right)$ if for every submodule $B$ of $M, N \cap B \leq Z_{2}(M)$ implies that $B \leq Z_{2}(M)$. It is clear that every essential submodule is t-essential, but not conversely. However, the two concepts are equivalent under the class of nonsingular modules. A submodule $N$ of $M$ is called fully invariant if $f(N) \leq N$ for every $R$-endomorphism $f$ of $M$.Clearly 0 and $M$ are fully invariant submodules of $M$ [15] . $M$ is called duo module if every submodule of $M$ is fully invariant. A submodule $N$ of an $R$-module is called stable if for each homomorphism f: $N \rightarrow M, f(N) \leq N$. A module is called fully stable if every submodule of $M$ is stable[1]. Asgari and Haghany [3] introduced the notion of $t$-semisimple modules as a generalization of semisimple modules. A module $M$ is $t$-semisimple if for every submodule $N$ of $M$, there exists a direct summand $K$ such that $K \leq_{\text {tes }} N$. In this paper we introduce the notion of strongly $t$-semisimple modules as a generalization of $t$-semisimple modules. An $R$-module is called strongly $t$-semisimple if for each submodule $N$ of $M$ there exists a fully invariant direct summand $K$ such that $K \leq_{\text {tes }} N$. It is clear that the class of strongly $t$-semisimple modules contains the class of $t$-semisimple. This paper consists of three sections. In Section 2 we introduce the concept of strongly $t$-semisimple and giving many characterizations and properties of this class of modules.

Section 3 , concerns with strongly $t$-semisimple rings. Several, characterization of commutative strongly $t$-semisimple ring. Also we give some characterizations of nonsingular strongly $t$-semisimple ring. First, we list some known result, which will be needed in our work.

Proposition 1.1. (see [2]) The following statements are equivalent for a submodule $A$ of an $R$-module.

(1) $A$ is $t$-essential in $M$;

(2) $\left(A+Z_{2}(M)\right) / Z_{2}(M)$ is essential in $M / Z_{2}(M)$;

(3) $A+Z_{2}(M)$ is essential in $M$;

(4) $M / A$ is $Z_{2}$-torsion.

Lemma 1.2. (see [4]) Let $A_{\lambda}$ be submodule of $M \lambda$ for all $\lambda$ in a set $\Lambda$. 
(1) If $\Lambda$ is a finite and $A_{\lambda} \leq_{\text {tes }} M_{\lambda}$, then $\bigcap_{\Lambda}\left|A_{\lambda} \leq_{\text {tes }} \bigcap_{\Lambda}\right| M_{\lambda}$ for all $\lambda \in \Lambda$.

(2) $\oplus_{\Lambda} A_{\lambda} \leq_{\text {tes }} \oplus \Lambda M_{\lambda}$ If and only if $A_{\lambda} \leq_{\text {tes }} M_{\lambda}$ for all $\lambda \in \Lambda$.

Lemma 1.3. (see [13]) Let $R$ be a ring and let $L \leq K$ be submodules of an $R$-module $M$ such that $L$ is a fully invariant submodule of $K$ and $K$ is a fully invariant submodule of $M$. Then $L$ is a fully invariant submodule of $M$.

Theorem 1.4. (see [3]) The following statements are equivalent for a module $M$ :

(1) $M$ is t-semisimple;

(2) $\left.M / Z_{2}(M)\right)$ is semisimple;

(3) $M=Z_{2}\left(M \oplus M^{\prime}\right)$ where $M^{\prime}$ is a non-singular semisimple module;

(4) Every nonsingular submodule of $M$ is a direct summand;

(5) Every submodule of $M$ which contains $Z_{2}(M)$ is a direct summand.

\section{Strongly t-Semisimple Modules}

Definition 2.1. An R-module is called strongly t-semisimple if for each submodule $\mathrm{N}$ of $\mathrm{M}$ there exists a fully invariant direct summand $\mathrm{K}$ such that $K \leq_{\text {tes }} N$.

Remarks and Examples. (1) It is clear that every strongly t-semisimple module is t-semisimple, but the convers is not true as we shall see later.

(2) If $\mathrm{M}$ is $Z_{2}$-torsion, then $\mathrm{M}$ is strongly t-semisimple.

Proof. Since $\mathrm{M}$ is $Z_{2}$-torsion, $Z_{2}(M)=M$. So that for all $A \leq M, Z_{2}(A)$ $=Z_{2}(M) \bigcap A=M \bigcap A=A$, then $(0)+Z_{2}(A)=A \leq_{\text {ess }} A$, Thus $(0) \leq_{\text {tes }} A$ for all $A \leq M$.But (0) is a direct summand of $M$, and (0) is fully invariant. Hence $M$ is strongly t-semisimple.

(3) Every singular module is strongly t-semisimple.

Proof. Let $M$ be a singular R-module. Then $Z(M)=M$, it follows that $Z_{2}(M)=Z(M)=M$. Thus $\mathrm{M}$ is $Z_{2}$ torsion, hence $M$ is strongly t-semisimple. Thus, in particular $Z_{n}$ as Z-module is strongly t-semisimple for all $n \in Z_{+}$, $n>1$.

(4) The converse of (3) is not true in general, for example $Z_{4}$ as $Z_{4}$-module is not singular, but it is $Z_{2}$-torsion, so it is strongly t-semisimple.

(5) If $M$ is t-semisimple module and weak duo (SS-module). Then M is strongly t-semisimple, where $\mathrm{M}$ is a weak duo(or SS-module) if every direct summand of $\mathrm{M}$ is fully invariant. 
Proof. Let $N \leq M$, since $M$ is t-semisimple, there exists $K \leq^{\oplus} M$ such that $K \leq_{\text {tes }} N$. But $M$ is $S S$-module, so $K$ is stable; hence $K$ is fully invariant direct summand. Thus $M$ is strongly t-semisimple, where $M$ is a weak duo(or $S S$-module) if every direct summand of $\mathrm{M}$ is fully invariant.

(6) If $M$ is t-semisimple and duo (or fully stable), then $M$ is strongly tsemisimple.

Hence every t-semisimple multiplication R-module is strongly t-semisimple.

(7) If $M$ is cyclic t-semisimple module over commutative $\operatorname{ring} R$ then $M$ is a strongly t-semisimple.

Proof. Since $M$ is cyclic module over commutative ring, then $M$ is a multiplication module. Thus $M$ is duo. Therefor the result follows by part (9).

(8) $M=Z_{n} \oplus Z$ as $Z$-module is not t-semisimple. For all $n \in Z_{+}, n>1$.

Proof. Suppose $M$ is t-semisimple. Then $M / Z_{n} \cong Z$ is t-semisimple [4, Corollary 2.4] which is a contradiction.

(9) t-semisimple module need not be strongly t-semisimple, for example:

Example 2.2. Let $T=M \oplus M$ where $\mathrm{M}$ is a non-singular semisimple R-module, $M \neq(0)$. Hence $T$ is semisimple, and so $T$ is a t-semisimple, let $N=M \oplus(0)$, so there exists $K \leq^{\oplus} M$ such that $K \leq_{\text {tes }} N$. Hence $K=K_{1} \oplus(0)$ for some $K_{1} \leq M$, if $K_{1}=(0)$, then $K=\left\langle(0,0)>\right.$ and $K \leq_{\text {tes }}^{\oplus} M \oplus(0)$. But $<(0,0)>+Z_{2}(M+(0)) \leq_{\text {ess }} M \oplus(0)$ (by Proposition 1.1.(3)) Thus $Z_{2}(M) \leq_{\text {ess }} M$. But $Z_{2}(M)=(0)$, hence $(0) \leq_{\text {ess }} M$ and so $M=(0)$, which is a contradiction. It follows that $K_{1} \neq(0)$, so $K \neq<(0,0)>$. But in this case $K$ is not fully invariant submodule of $\mathrm{T}$. To see this:

Let $f: T \rightarrow T$ defined by $T(x, y)=(y, x)$, for all $(x, y) \in T$, Then $T\left(K_{1} \oplus\right.$ $(0))=(0) \oplus K_{1} \leq \neq K_{1} \oplus(0)$. Thus $K=K_{1} \oplus(0)$ is not fully invariant submodule of $\mathrm{T}$, such that $K \leq_{\text {tes }} \mathrm{N}$. Therefore $\mathrm{T}$ is not strongly t-semisimple.

In particular, $\mathrm{R}$ as $\mathrm{R}$-module is simple non-singular $\mathrm{R}$-module, so $R \oplus R$ as $\mathrm{R}$-module is semisimple and so it is t-semisimple .But $R \oplus R$ is not strongly t-semisimple:

To see this, let $N=R \oplus(0)$. As $<(0,0)>$ is only direct summand fully invariant of $R \oplus R$, such that $<(0,0)>\leq N=R(0)$. But $<(0,0)>\leq \neq 2010_{\text {tes }}$ $N$ because if we assume that $<(0,0)>\leq_{\text {tes }} N$ then $<(0,0)>+Z_{2}(N) \leq_{\text {ess }} N$, so that $\langle(0,0)\rangle+<(0,0)\rangle=<(0,0)>\leq_{e s s} N$ which is a contradiction.

Now we shall give some characterizations of strongly t-semisimple.

Theorem 2.3. The following statements are equivalent for an $R$-module M: 
(1) $M$ is strongly t-semisimple, of $M$,

(2) $\frac{M}{Z_{2}(M)}$ is fully stable semisimple and isomorphic to a stable submodule

(3) $M=Z_{2}(M) \oplus M^{\prime}$ where $M^{\prime}$ is a nonsingular semisimple fully stable module and $M^{\prime}$ is a stable submodule in $M$,

(4) Every nonsingular submodule of $M$ is stable direct summand,

(5) Every submodule of $M$ which contains $Z_{2}(M)$ is a direct summand of $M$ and $\frac{M}{Z_{2}(M)}$ is fully stable and isomorphic to a stable submodule of $M$.

Proof. $(1) \Rightarrow(4)$ Let $\mathrm{N}$ be a nonsingular submodule of M. Since M is strongly t-semisimple, there exists a fully invariant direct summand $\mathrm{K}$ of $\mathrm{M}$ such that $K \leq_{\text {tes }} N$. Assume that $M=K \oplus K^{\prime}$ for some $K^{\prime} \leq M$.Hence $N=\left(K \oplus K^{\prime}\right) \bigcap N$ and so $N=K \oplus\left(K^{\prime} \cap N\right)$ by modular law. Thus $K \leq^{\oplus} N$ and $\frac{N}{K} \cong\left(N \bigcap K^{\prime}\right)$. But $K \leq_{\text {tes }} N$ implies $\frac{N}{K}$ is $Z_{2}$-torsion that is $Z_{2}\left(\frac{N}{K}\right)=\frac{N}{K}$ by Proposition (1.1). On the other hand $\left(N \cap K^{\prime}\right) \leq N$ and $\mathrm{N}$ is nonsingular, so $\left(N \cap K^{\prime}\right)$ is nonsingular submodule, and hence $\frac{N}{K}$ is nonsingular, which implies that $Z_{2}\left(\frac{N}{K}\right)=0$.Thus $\frac{N}{K}=0$ and hence $N=K$. Therefore $\mathrm{N}$ is a fully invariant direct summand, and hence $\mathrm{N}$ is a stable direct summand.

$(4) \Rightarrow(3)$ Let $M^{\prime}$ be a complement of $Z_{2}(M)$. Hence $M^{\prime} \oplus Z_{2}(M) \leq{ }_{\text {ess }} M$ And so $M^{\prime} \leq_{\text {tes }} M$ by Proposition (1.1(3)).Thus $\frac{M}{M^{\prime}}$ is $Z_{2}$-torsion, by proposition (1.1(4)). We claim that $M^{\prime}$ is nonsingular. To explain our assertion, suppose $x \in Z\left(M^{\prime}\right)$, so $x \in M^{\prime} \leq M$ and $\operatorname{ann}(x) \leq_{\text {ess }} R$. Hence $\operatorname{ann}(x) \leq_{\text {tes }} R$ and this implies $x \in Z_{2}(M)$. Thus $x \in Z_{2}(M) \bigcap M^{\prime}=(0)$, thus $\mathrm{x}=0$ and $M^{\prime}$ is a nonsingular. So that by hypothesis, $M^{\prime}$ is a stable direct summand of $\mathrm{M}$ and so that $M=L \oplus M^{\prime}$ for some $L \leq M$.Thus $L \cong \frac{M}{M^{\prime}}$ which is $Z_{2}$-torsion, hence $\mathrm{L}$ is $Z_{2}$-torsion . On other hand, $Z_{2}(M)=Z_{2}\left(M^{\prime}\right)+Z_{2}(L)=0+L=L$. It follows that $M=Z_{2}(M) \oplus M^{\prime}, M^{\prime}$ is a nonsingular. Now let $N \leq M^{\prime}$, so $\mathrm{N}$ is a nonsingular and hence $N \leq{ }^{\oplus} M$ by hypothesis. It follows that $M=N \oplus W$ for some $W \leq M$ and hence $M^{\prime}=(N \oplus W) \bigcap M^{\prime}$ and so $M^{\prime}$ $=N \oplus\left(W \cap M^{\prime}\right)$ by modular law. Thus $N \leq{ }^{\oplus} M^{\prime}$ and hence $M^{\prime}$ is semisimple .Next to prove $M^{\prime}$ is fully stable. It is sufficient to prove that every submodule of $M^{\prime}$ is fully invariant, so let $N \leq M^{\prime} \leq M$ and let $f: M^{\prime} \rightarrow M^{\prime}$. Then $i \circ f \circ \rho \in \operatorname{End}(M)$, where i inclusion map from $M^{\prime}$ to $\mathrm{M}$ and $\rho$ is the projection of M onto $M^{\prime}$. Then $(i \circ f \circ \rho)(N) \leq N$ since $\mathrm{N}$ is stable in M (by hypothesis). Now $(i \circ f \circ \rho)(N)=\left(i \circ f(\rho(N))\right.$, but $N \leq M^{\prime}$, so $\rho(N)=N$. Thus $i \circ f(\rho(N))=i \circ f(N)=f(N) \leq N$. Thus $\mathrm{N}$ is fully invariant submodule of $M^{\prime}$, but $N \leq^{\oplus} M$, so that $\mathrm{N}$ is stable in $M^{\prime}$ and $M^{\prime}$ is fully stable.

$(3) \Rightarrow(1)$ Let $M=Z_{2}(M) \oplus M^{\prime}, M^{\prime}$ is nonsingular semisimple fully stable 
module, $M^{\prime}$ is stable in $\mathrm{M}$. Let $N \leq M$, then $\left(N \cap M^{\prime}\right) \leq M^{\prime}$, so $\left(N \cap M^{\prime}\right.$ )$\leq{ }^{\oplus} M^{\prime}$ (since $M^{\prime}$ is semisimple). It follows that $M^{\prime}=\left(N \cap M^{\prime}\right) \oplus W$ for some $W \leq M^{\prime}$ and hence $M=Z_{2}(M) \oplus\left(N \cap M^{\prime}\right) \oplus W$. Hence $\left(N \cap M^{\prime}\right) \leq{ }^{\oplus} M$. On other hand, $\frac{N}{N \cap M^{\prime}} \cong \frac{N+M^{\prime}}{M^{\prime}} \leq \frac{M}{M^{\prime}} \cong Z_{2}(M)$. But $Z_{2}(M)$ is $Z_{2}$-torsion. Hence, $\frac{N}{N \cap M^{\prime}}$ is $Z_{2}$-torision and then by (Proposition 1.1(4) $\left(N \cap M^{\prime}\right) \leq_{\text {tes }} N$. But $\left(N \cap M^{\prime}\right)$ is stable in $M^{\prime}$ (since $M^{\prime}$ is fully stable) so $N \cap M^{\prime}$ is a fully invariant submodule in $\mathrm{M}$. Thus by Lemma (1.3) $N \cap M^{\prime}$ is fully invariant in M. But $N \cap M^{\prime}$ is direct summand of M. Thus $N \cap M^{\prime} \leq{ }^{\oplus} M, N \cap M^{\prime} \leq N$, hence $\mathrm{M}$ is strongly t-semisimple.

$(3) \Rightarrow(5)$ Let $N \leq M, N \supseteq Z_{2}(M)$. Since $M=Z_{2}(M) \oplus M^{\prime}$, where $M^{\prime}$ is a nonsingular semisimple fully stable, $M^{\prime}$ is stable in M.Then $N=\left(Z_{2}(M) \oplus M^{\prime}\right.$ )$\bigcap N=Z_{2}(M) \oplus\left(N \cap M^{\prime}\right)$ by modular law. But $\left.N \cap M^{\prime}\right) \leq M^{\prime}$ and $M^{\prime}$ is semisimple implies $\left(N \cap M^{\prime}\right) \leq{ }^{\oplus} M^{\prime}$. It follows that $\left(N \cap M^{\prime}\right) \oplus W=M^{\prime}$. Hence $M=Z_{2}(M) \oplus\left(N \cap M^{\prime}\right) \oplus W=N \oplus W$. Thus $N \leq{ }^{\oplus} M$. Also $\frac{M}{\left(Z_{2}(M)\right)} \cong$ $M^{\prime}$ and $M^{\prime}$ is a fully stable module and $M^{\prime}$ is stable in $\mathrm{M}$, so that $\frac{M}{\left(Z_{2}(M)\right.}$ is fully stable semisimple and isomorphic to stable submodule of M.

$(2) \Rightarrow(3)$ Since $Z_{2}(M)$ is t-closed, $\frac{M}{\left(Z_{2}(M)\right)}$ is nonsingular. By condition (2), $\frac{M}{\left(Z_{2}(M)\right)}$ is semisimple, hence $\frac{M}{\left(Z_{2}(M)\right)}$ is projective (by [10, Coroallary 1.25,P.35] . Now let $\left.\pi: M \rightarrow M /\left(Z_{(} 2\right)(M)\right)$ be the natural epiomorphism and as $\frac{M}{\left(Z_{2}(M)\right)}$ is projective, we get $k e r \pi=Z_{2}(M)$ is a direct summand of $\mathrm{M}$.Hence $M=$ $Z_{2}(M) \oplus M^{\prime}$. Thus $M^{\prime} \cong \frac{M}{\left(Z_{2}(M)\right)}$ which is nonsingular semisimple fully stable module. Then $M^{\prime}$ is nonsingular semisimple fully stable. Also $M^{\prime}$ is stable submodule of $\mathrm{M}$ by condition (2).

(3) $\Rightarrow(2)$ By condition (3), $M=Z_{2}(M) \oplus M^{\prime}$, where $M^{\prime}$, is a nonsingular semisimple fully stable module and $M^{\prime}$ is stable in M. It follows that $\frac{M}{\left(Z_{2}(M)\right)} \cong$ $M^{\prime}$. Thus $\frac{M}{\left(Z_{2}(M)\right)}$ is semisimple fully stable and isomorphic to stable submodule $M^{\prime}$ of $\mathrm{M}$.

$(2) \Rightarrow(5)$ It follows directly (since $(2) \Leftrightarrow(3) \Rightarrow(5)$ then $(2) \Rightarrow(5)$ ).

(5) $\Rightarrow(2)$ Let $\frac{N}{Z_{2}(M)} \leq \frac{M}{Z_{2}(M)}$. Then $N \supseteq Z_{2}(M)$, so by condition (5), N is stable direct summand of $\mathrm{M}$, so that $N \oplus W=M$ for some $W \leq M$. Thus $\frac{N}{\left(Z_{2}(M)\right)}+\frac{\left(W+Z_{2}(M)\right)}{\left(Z_{2}(M)\right)}=\frac{M}{\left(Z_{2}(M)\right)}$. But we can show that $\frac{N}{\left(Z_{2}(M)\right)} \bigcap \frac{\left(N+\overline{Z_{2}}(M)\right)}{\left(Z_{2}(M)\right)}=0$, as follows:

Let $\bar{x} \in \frac{N}{\left(Z_{2}(M)\right)} \cap \frac{\left(W+Z_{2}(M)\right)}{\left(Z_{2}(M)\right)}$. Then $\bar{x}=n+Z_{2}(M)=w+Z_{2}(M)$ for some $n \in N, w \in W$, and so $n-w \in Z_{2}(M) \subseteq N$. It follow that $n-w=n_{1}$ for 
some $n_{1} \in N$ and hence $n-n_{1}=w \in N \cap W=0$. Thus $x=0_{\frac{M}{Z_{2}(M)}}$ and so $\frac{N}{\left(Z_{2}(M)\right)} \oplus \frac{\left(W+Z_{2}(M)\right)}{Z_{2}(M)}=\frac{M}{\left(Z_{2}(M)\right)}$. This implies $\frac{M}{Z_{2}(M)}$ is semisimple. By condition (5), $\frac{M}{\left(Z_{2}(M)\right)}$ fully stable and isomorphic to stable submodule of M . But $\frac{M}{Z_{2}(M)}$ is nonsingular, so $\frac{M}{Z_{2}(M)}$ is projective and hence $M=Z_{2}(M)+M^{\prime}$. Thus $M^{\prime}$ is nonsingular semisimple (since $M^{\prime} \cong \frac{M}{Z_{2}(M)}$ ). It follows that $M^{\prime}$ is fully stable module and $M^{\prime}$ is stable in $\mathrm{M}$.

Now we shall give some other properties of strongly t-semisimple.

Recall that an R-module $\mathrm{M}$ is called quasi-Dedekind if $\operatorname{Hom}\left(\frac{M}{N}, M\right)=0$ for all nonzero submodule $\mathrm{N}$ of M.Equivantally, $\mathrm{M}$ is quasi-Dedkind if for each $f \in \operatorname{End}(M), f \neq 0$, then $\operatorname{ker} f=0[10]$

Proposition 2.4. If $M$ is a quasi-Dedekind module, then $M$ is t-semisimple if and only if $M$ is strongly t-semisimple.

Proof. $\Rightarrow$ since $\mathrm{M}$ is quasi-Dedekind, then for each $f \in E n d M f \neq 0$, $\operatorname{Kerf}=0$, and hence kerf is stable and so that by [14], M is SS-module and so that $\mathrm{M}$ is strongly t-semisimple by Remarks and Examples 2.2(8).

$\Leftarrow$ It is clear.

To prove the next result, we state and prove the following Lemma.

Lemma 2.5. Let $N$ be a submodule of $M$ and $K$ is a direct summand of $M$ such that $K \leq N$. If $K$ is fully invariant submodule in $M$, then $K$ is a fully invariant submodule in $N$.

Proof. To prove $\mathrm{K}$ is a fully invariant submodule of N. Let $\varphi: N \rightarrow N$ be an R-homomorphism, to prove $\varphi(K) \leq K$.

Consider the sequence $M \stackrel{\rho}{\rightarrow} K \stackrel{i n c}{\rightarrow} N \stackrel{\varphi}{\rightarrow} N \stackrel{j}{\rightarrow} M$. Where $\rho$ is the natural projection and i,j are the inclusion mapping. Then $(j \circ \varphi \circ i \circ \rho) \in E n d M$, and since $\mathrm{K}$ is a fully invariant in $\mathrm{M}$, so $(j \circ \varphi \circ i \circ \rho)(K) \subseteq K$. But $j \circ \varphi(\rho(K))=j \circ$ $\varphi(K)=\varphi(K)$, hence $\varphi(K) \leq K$. Thus $\mathrm{K}$ is a fully invariant submodule of $\mathrm{N}$.

Proposition 2.6. Every submodule of strongly t-semisimple module is strongly t-semisimple.

Proof. Let $N \leq M$, let $W \leq N$, so $W \leq M$. Since M is strongly tsemisimple, there exists fully invariant direct summand $\mathrm{K}$ of $\mathrm{M}$ such that $K \leq_{\text {tes }}$ $W \leq N$. As $K \leq{ }^{\oplus} M, M=K \oplus K^{\prime}$ for some $K^{\prime} \leq M$ then, $N=N \bigcap\left(K \oplus K^{\prime}\right.$ )$=K \oplus\left(K^{\prime} \cap \cap N\right)$. So that $K \leq^{\oplus} N$, and by Lemma (2.5) $\mathrm{K}$ is fully invariant submodule of $\mathrm{N}$. Therefore, $\mathrm{K}$ is fully invariant direct summand of $\mathrm{N}$ such that $K \leq_{\text {tes }} W \leq N$. Thus $\mathrm{N}$ is a strongly t-semisimple module.

Now we consider the direct sum of strongly t-semisimple. First we no- 
tice that direct sum of strongly t-semisimple module need not be strongly tsemisimple for example:

Consider $\mathrm{R}$ as $\mathrm{R}$-module $\mathrm{R}$ is strongly t-semisimple. But $M=R \oplus R$ is not strongly t-semisimple by Remarks and Examples 2.2(12). However, the direct sum of strongly t-semisimple is strongly t-semisimple under certain condition. Before giving our next result, we present the following lemma.

Lemma 2.7. Let $M=M_{1} \oplus M_{2}$ such that ann $M_{1}+$ ann $M_{2}=R$.Then $\operatorname{Hom}\left(M_{1}, M_{2}\right)=0$ and $\operatorname{Hom}\left(M_{2}, M_{1}\right)=0$.

Proof. since $R=$ ann $M_{1}+$ ann $M_{2}$, then $M_{1}=M_{1}\left(a n n M_{1}\right)+M_{1}\left(\right.$ ann $\left.M_{2}\right)$. Put ann $M_{1}=A_{1}$, ann $M_{2}=A_{2}$, therefore $M_{1}=M_{1} A_{1}+M_{1} A_{2}=M_{1} A_{2}$, then for each $\varphi \in \operatorname{Hom}\left(M_{1}, M_{2}\right), \varphi\left(M_{1}\right)=\varphi\left(A_{2} M_{1}\right)=\varphi\left(M_{1}\right) A_{2} \leq M_{2} A_{2}=0$, hence $\varphi=0$.Thus $\operatorname{Hom}\left(M_{1}, M_{2}\right)=0$. Similarly, $\operatorname{Hom}\left(M_{2}, M_{1}\right)=0$.

Theorem 2.8. Let $M=M_{1} \oplus M_{2}$ such that ann $M_{1}+$ ann $M_{2}=R$. Then $M_{1}, M_{2}$ are strongly t-semisimple if and only if $M=M_{1} \oplus M_{2}$ is strongly t-semisimple.

Proof. $\Leftarrow$ By Proposition(2.6)

$\Rightarrow$ Let $N \leq M$. Since ann $M_{1}+$ ann $M_{2}=R, N=N_{1} \oplus N_{2}$ for some $N_{1}$ and $N_{2}$ submodules of $M_{1}$ and $M_{2}$ respectively. As $M_{1}$ and $M_{2}$ are strongly t-semisimple, then there exist $K_{1} \leq M_{1}$ and $K_{2} \leq M_{2}$ such that $K_{1}$ is a direct summand of $M_{1}, K_{1}$ is fully invariant in $M_{1}$ and $K_{1}$ is t-essential in $N_{1}, K_{2}$ is a direct summand of $M_{2}, K_{2}$ is fully invariant in $M_{2}$ and $K_{2}$ is t-essential in $N_{2}$. But $K_{1} \leq^{\oplus} M_{1}$ and $K_{2} \leq^{\oplus} M_{2}$ imply $K_{1} \oplus K_{2} \leq^{\oplus} M_{1} \oplus M_{2}$ and $K_{1} \leq_{\text {tes }} N_{1}$, $K_{2} \leq_{\text {tes }} N_{2}$ imply $K_{1} \oplus K_{2} \leq_{\text {tes }} N_{1} \oplus N_{2}$ by Proposition (1.2).

Now, let

$$
\begin{aligned}
\varphi \in \operatorname{End}\left(M_{1}, M_{2}\right) \cong\left(\begin{array}{cc}
\operatorname{End} M_{1} & \operatorname{Hom}\left(M_{2}, M_{1}\right) \\
\operatorname{Hom}\left(M_{1}, M_{2}\right) & \text { End } M_{2}
\end{array}\right) \\
\quad=\left(\begin{array}{cc}
\operatorname{End} M_{1} & 0 \\
0 & \text { End } M_{2}
\end{array}\right)
\end{aligned}
$$

so

$$
\varphi=\left(\begin{array}{cc}
\varphi_{1} & 0 \\
0 & \varphi_{2}
\end{array}\right)
$$

for some $\varphi_{1} \in$ End $M_{1}, \varphi_{2} \in$ End $M_{2}$. Then $\varphi\left(K_{1} \oplus K_{2}\right)=\varphi_{1}\left(K_{1}\right) \oplus \varphi_{2}\left(K_{2}\right)$ $\leq K_{1} \oplus K_{2}$ since $K_{1}$ is fully invariant in $M_{1}$ and $K_{2}$ is fully invariant in $M_{2}$. Hence $\mathrm{M}$ is strongly t-semisimple.

Now we shall give other characterizations of strongly t-semisimple module. 
Proposition 2.9. The following statements are equivalent for a module $M$, such that any direct summand has a unique complement:

(1) $M$ is strongly t-semisimple,

(2) For each submodule $N$ of $M$, there exists a decomposition $M=K \oplus L$ such that $K \leq N$ and $L$ is stable in $M$ and $N \cap L \leq Z_{2} L$,

(3) For each submodule $N$ of $M, N=K \oplus K^{\prime}$ such that $K$ is a direct summand stable in $M$ and $K^{\prime}$ is $Z_{2}$-torsion.

Proof. (1) $\Rightarrow(2)$

Let $\mathrm{K}$ be a complement of $Z_{2}(N)$ in $\mathrm{N}$. Then $K+Z_{2}(N) \leq_{\text {ess }} N$ and let $\mathrm{C}$ be a complement of $K \oplus Z_{2}(M)$. So $K \oplus Z_{2}(M) \oplus C \leq_{e s s} M$ and hence $K \oplus Z_{2}(M) \oplus C \leq_{\text {tes }} M$. But $\mathrm{M}$ is strongly t-semisimple implies $\mathrm{M}$ t-semisimple, hence $K \oplus Z_{2}(M) \oplus C=\mathrm{M}$ ( by [4,Corollary 2.7].Put $Z_{2}(M) \oplus C=\mathrm{L}$. Then $M=K \oplus L$ and hence $N=(K \oplus L) \bigcap N=K \oplus(N \cap L)$ (by modular law ). But $K+Z_{2}(N) \leq_{\text {ess }} N$ implies $\frac{N}{K}$ is $Z_{2}$-torsion (by Proposition (1.1)). On other hand, $\frac{N}{K} \cong N \cap L$, so that $N \cap L$ is $Z_{2}$-torsion. Thus $N \cap L=Z_{2}(L \bigcap N) \leq Z_{2}(L)$. Now, $\mathrm{C}$ is a complement of $K \oplus Z_{2}(M)$ which is a direct summand of $\mathrm{M}$, and by hypothesis, $\mathrm{C}$ is a unique complement and hence by [2, Theorem(1.4.8)] $\mathrm{C}$ is stable and hence $L=Z_{2}(M) \oplus C$ is stable submodule in M. Thus $M=K \oplus L$ is the desired decomposition.

$(2) \Rightarrow(3)$ By condition (2) $M=K \oplus L$ such that $K \leq N$, L is stable and $N \bigcap L \leq Z_{2}(L)$. Hence $N=(K \oplus L) \bigcap N=K \oplus(L \bigcap N)$, put $K^{\prime}=L \bigcap N$, so $N=K \oplus K^{\prime}, \frac{N}{K} \cong K^{\prime}=L \bigcap N$ is $Z_{2}$-torsion, $\mathrm{K}$ is stable in $\mathrm{M}$ (since $\mathrm{K}$ is complement of $\mathrm{L}$ which is direct summand of $\mathrm{M}$ ).

$(3) \Rightarrow(1)$ By condition (3), N $=K \oplus K^{\prime}, K \leq^{\oplus} M$ and $\mathrm{K}$ is stable in $\mathrm{M}$ and $K^{\prime}$ is $Z_{2}$-torsion. Then $K \leq{ }^{\oplus} M$ and $K \leq N$ and $\frac{N}{K} \cong K^{\prime}$ is $Z_{2}$-torsion. Hence $K \leq_{\text {tes }} N$ and so that $\mathrm{M}$ is strongly t-semisimple.

Definition 2.10. (see [7]) An R-module $\mathrm{M}$ is called comultiplication if $\operatorname{ann}_{M} a n n_{R} N=N$ for every submodule $\mathrm{N}$ of $\mathrm{M}$.

Lemma 2.11. Every comultiplication module is fully stable.

Proof. Let $\mathrm{M}$ be a comultiplication R-module. Then $\operatorname{ann}_{M} a n n_{R} N=N$ for all $N \leq M$. Hence $\operatorname{ann}_{M} \operatorname{ann}_{R}(x R)=x R$ for all cyclic submodules $x R$ in $M$.Thus $M$ is fully stable, [2, Corollary $(3.5)]$.

Corollary 2.12. Let $M$ be a comultiplication $R$-module. Then $M$ is t-semisimple if and only if $M$ is strongly t-semisimple.

Proof. $\Leftarrow$ It is clear.

$\Rightarrow$ It follows directly by Lemma (2.11) and Remarks and Examples 2.2(6). 
Recall that an R-module $\mathrm{M}$ is called a principally injective if for any $a \in R$, any homomorphism $f: R a \rightarrow M$ extends to an R-homomorphism from $R_{R}$ to $\mathrm{M}[12]$.

Corollary 2.13. Let $M$ be a principally injective. Then $M$ is t-semisimple if and only if $M$ strongly t-semisimple.

Proof. $\Leftarrow$ It is clear.

$\Rightarrow \mathrm{M}$ is principally injective implies that $\operatorname{ann}_{M} \operatorname{ann}_{R}(x)=(x)$ for each $x \in R$. Hence by [2, Corollary(3.5)] $\mathrm{M}$ is fully stable. Then by Remark and Examples 2.2(5), $\mathrm{M}$ is strongly t-semisimple.

Corollary 2.14. (2.14):

$M$ is injective $R$ - module. Then $M$ is t-semisimple $R$ - module if and only if $M$ is strongly t-semisimple.

Definition 2.15. (2.15) [12]:

An R-module is called scalar if for all $\varphi \in \operatorname{End} M$, there exists $r \in R$ such that $\varphi(x)=x r$ for all $x \in M$, where $\mathrm{R}$ is a commutative ring.

Proposition 2.16. (2.16):

Let $M$ be a scalar $R$-module. Then $M$ is t-semisimple if and only if $M$ is strongly t-semisimple, where $R$ is commutative.

Proof: $\Leftarrow$ It is clear.

$\Rightarrow$ Let $\mathrm{N} \leq M$, let $\varphi \in$ End $M$. Since $\mathrm{M}$ is scalar, there exists $r \in R$ such that $\varphi(x)=x r$,for all $x \in M$. Hence $\varphi(N)=N r \leq N$ and so that $\mathrm{N}$ is fully invariant submodule. Thus $\mathrm{M}$ is duo. But $\mathrm{M}$ is duo and t-semisimple implies $\mathrm{M}$ is strongly t-semisimple by Remarks and Examples 2.2(6).

Proposition 2.17. (2.17):

Let $M$ be a duo $R$-module. Then the following statements are equivalent

(1) Every $R$-module is t-semisimple and $Z_{2}(M)$ is projective.

(2) Every $R$-module is strongly t-semisimple and $Z_{2}(M)$ is projective.

(3) $R$ is semisimple.

Proof: $(1) \Rightarrow(3)$

Let $\mathrm{M}$ be an $\mathrm{R}$-module. Then $\mathrm{M}$ is t-semisimple by hypothesis. Hence $M=$ $Z_{2}(M) \oplus M^{\prime}$, where $M^{\prime}$ is a nonsingular semisimple. It follows that $M^{\prime}$ is projective, but by hypothesis $Z_{2}(M)$ is projective. Thus $\mathrm{M}$ is projective, that is every R-module is projective and so by [11, Corollary $8.2 .2(\mathrm{e})] \mathrm{R}$ is semisimple. $(3) \Rightarrow(1)$

Since $\mathrm{R}$ is semisimple, every R-module is semisimple by [11, Corollary 8.2.2(a)] 
Hence every $\mathrm{R}$-module is t-semisimple. Also $\mathrm{R}$ is semisimple, then every $\mathrm{R}$ module is projective [11, Corollary 8.2.2(e)]. Thus $Z_{2}(M)$ is projective.

$(1) \Rightarrow(2)$ It follows by Remark and Examples (2.2.(6))

$(2) \Rightarrow(1)$ It is clear.

Proposition 2.18. (2.18):

Let $M$ be a duo $R$-module if $R$ is semisimple then every $R$-module is strongly $t$-semisimple, and conversely hold if $R$ is nonsingular.

Proof: $\Rightarrow \mathrm{R}$ is semisimple implies every $\mathrm{R}$-module $\mathrm{M}$ is semisimple and hence t-semisimple. But $\mathrm{M}$ is duo by hypothesis, so that $\mathrm{M}$ is strongly tsemisimple by Remark and Examples 2.2(6).

$\Leftarrow$ By hypothesis, $\mathrm{R}$ is t-semisimple. But $\mathrm{R}$ is nonsingular, so $\mathrm{R}$ is semisimple Now we introduce the following:

Definition 2.19. (2.19):

An R-module $\mathrm{M}$ is called t-uniform if every submodule of $\mathrm{M}$ is t-essential.

Proposition 2.20. (2.20):

If $M$ is t-uniform then $M$ is strongly t-semisimple.

Proof: Since M is t-uniform, $(0) \leq_{\text {tes }} \mathrm{M}$. Hence $\frac{M}{(0)}$ is $Z_{2}$-torsion (by proposition. 1.1(4)); that is $\mathrm{M}$ is $Z_{2}$-torsion (so $M=Z_{2}(M)$ ). Now for all $N \leq M$, $Z_{2}(N)=Z_{2}(M) \bigcap N=N$. Hence $(0) \leq_{\text {tes }} N\left(\right.$ since $(0)+Z_{2}(N)=0+N=$ $\left.N \leq_{\text {ess }} N\right)$.But (0) is fully invariant direct summand of $\mathrm{M}$.Thus $\mathrm{M}$ is strongly t-semisimple.

Remark 2.21. (2.21):

A uniform module need not be t-uniform.

Example 2.22. (2.22):

Consider Z- module $Z_{6}, Z_{6}$ is singular, hence $Z_{6}$ is $Z_{2}$-torsion; that is $Z_{2}\left(Z_{6}\right)=$ $Z_{6}$. Hence for each $N \leq Z_{6}, N+Z_{2}\left(Z_{6}\right)=Z_{6} \leq_{e s s} Z_{6}$ and then by Proposition (1.1), $N \leq_{\text {tes }} Z_{6}$. Thus $Z_{6}$ is t-uniform. But $Z_{6}$ is not uniform.

Remark 2.23. (2.23):

It is clear that t-uniform module need not uniform, as the following example shows.

Example 2.24. (2.24):

$\mathrm{Z}_{6}$ as $Z$-module $, \mathrm{Z}_{2}(M)=Z_{6}=M,(\overline{0}) \leq_{\text {tes }} M$ since $(\overline{0})+Z_{2}(M)=M \leq_{\text {ess }} M$, Let $N_{1}=<\overline{2}>\leq_{\text {tes }} M$ since $<\overline{2}>+Z_{2}(M)=M \leq_{\text {ess }} M$, similarly $N_{2}=<$ $\overline{3}>\leq_{\text {tes }} M, N_{3}=M \leq_{\text {tes }} M$. Thus $\mathrm{M}$ is t-uniform, but $\mathrm{M}$ is not uniform. 
Remark 2.25. (2.25):

$\mathrm{M}$ is t-uniform then $\frac{M}{N}$ is t-semisimple for all $N \leq M$.

Proof: For each $N \leq M, N \leq_{\text {tes }} M$. Then $\frac{M}{N}$ is $Z_{2}$-torsion (by proposition 1.1(4)). Hence $\frac{M}{N}$ is strongly t-semisimple by Remarks and Examples 2.2(2).

\section{Strongly t-Semisimple Rings}

Proposition 3.1. (3.1):

Every commutative $t$-semisimple ring $R$ is strongly $t$-semisimple ring $R$.

Proof: Since $\mathrm{R}$ is commutative ring, then $\mathrm{R}$ is duo $\mathrm{R}$-module and t-semisimple, implies $\mathrm{R}$ is strongly t-semisimple by Examples and Remarks 2.2(6).

Proposition 3.2. (3.2):

Let $R$ be a commutative Artinian ring with $\operatorname{Rad} R \leq_{\text {tes }} R$. Then $R$ is strongly $t$-semisimple. In particular every local Artinain ring is strongly $t$-semisimple.

Proof: By [4, Proposition 3.1 ], $\mathrm{R}$ is t-semisimple ring. Hence by Proposition (3.1), $\mathrm{R}$ is strongly t-semisimple.

Example 3.3. (3.3):

The ring $Z_{P \infty}$ is Artnian and $\operatorname{Rad} Z_{P^{\infty}}=Z_{P^{\infty}} \leq_{\text {ess }} Z_{P^{\infty}}$. Hence by Proposition (3.2), $Z_{P \infty}$ is strongly t-semisimple.

Proposition 3.4. (3.4):

The following statements are equivalent for a commutative ring

(1) $R$ is strongly t-semisimple;

(2) $R$ is t-semisimple;

(3) Every R-module is t-semisimple;

(4) Every nonsingular R-module is semisimple;

(5) Every nonsingular R-module is injective;

(6)Every $R$-module $M$ there is an injective submodule $M^{\prime}$ such that $M=Z_{2}$

$(M) \oplus M^{\prime}$

(7) $\frac{R}{Z_{2}(R)}$ is a semisimple ring.

(8) Every maximal ideal which contains $Z_{2}(R)$ is a direct summand;

(9) $R$ is a direct product of two ring, one is $Z_{2}$ - torsion and other is semisimple ring.

Proof: $(1) \Rightarrow(2)$ It is clear

$(2) \Rightarrow(1)$ It is follows by (Proposition 3.1). 
$(2) \Leftrightarrow(3) \Leftrightarrow(4) \Leftrightarrow(5) \Leftrightarrow(6) \Leftrightarrow(7)[3$, Theorem $(3.2)]$.

$(2) \Leftrightarrow(8) \Leftrightarrow(9)$ It follows by [3, Theorem 3.8]

Corollary 3.5. (3.5) [4]:

Let $R$ be a t-semisimple ring.

(1) A maximal right ideal I of $R$ is a direct summand if and only if it contains $Z_{2}(R)$.

(2) A minimal right ideal $J$ of $R$ is a direct summand if and only if it is nonsingular.

Corollary 3.6. (3.6):

Let $R$ be a strongly t-semisimple. A maximal ideal $I$ of $R$ is a direct summand if and only if $I \supseteq Z_{2}(R)$. A minimum ideal $I$ of $R$ is a direct summand if and only if $I$ is nonsingular.

Proof: It follows directly by (Corollary (3.5)).

Recall that a ring $\mathrm{R}$ is called quasi-Frobenius if $\mathrm{R}$ is self-injective and Noetherian.Equivalently " $\mathrm{R}$ is called quasi-Frobenius if $\mathrm{R}$ is self-injective and Artinian $[9]$.

Corollary 3.7. (3.7):

Let $R$ be a right nonsingular. Then $R$ is quasi-Frobenius if and only if $R$ is semisimple [3].

Proposition 3.8. (3.8):

Let $R$ be a nonsingular ring. Then the following statements are equivalent:

(1) $R$ is quasi-Frobenius;

(2) $R$ is semisimple;

(3) $R$ is t-semisimple ( $R$ is strongly t-semisimple);

(4) Every $R$-module is t-semisimple;

(5) Every nonsingular R-module is semisimple;

(6) Every nonsingular R-module is injective;

(7) For every $R$-module $M$, there exists an injective submodule $M^{\prime}$ such that $M=Z_{2}(M) \oplus M^{\prime}$;

(8) $\frac{R}{Z_{2}(R)}$ is a semisimple ring.

Proof: $(3) \Leftrightarrow(4) \Leftrightarrow(5) \Leftrightarrow(6) \Leftrightarrow(7) \Leftrightarrow(8)$ by Proposition (3.4).

$(1) \Leftrightarrow(2)$ It follows by Corollary $(3.7)$

$(2) \Leftrightarrow(3)$ It follows by [3] and Proposition(3.4).

Proposition 3.9. (3.9):

The following statements are equivalent for a commutative ring $R$ 
(1) $R$ is t-semisimple ( $R$ is strongly t-semisimple );

(2) Every weak duo module (SS-module) is strongly t-semisimple;

(3) Every $R$-module is t-semisimple.

Proof: $(1) \Leftrightarrow(3)$ by Proposition (3.4)

$(3) \Leftrightarrow(2)$ It follows by Remarks Examples 2.5(8).

$(2) \Rightarrow(1) \mathrm{R}$ is duo (because $\mathrm{R}$ is commutative ring with unity), so $\mathrm{R}$ is strongly t-semisimple.

Proposition 3.10. (3.10):

The following statements are equivalent for a commutative ring $R$ :

(1) $R$ is t-semisimple;

(2) Every nonsingualr $R$-module is strongly t-semisimple;

(3) For every $R$-module $M$, there exists a strongly t-semisimple $R$-module $M^{\prime}$ such that $M=Z_{2}(M) \oplus M^{\prime}$.

Proof: $(1) \Rightarrow(2)$ Let $\mathrm{M}$ be a nonsingular $\mathrm{R}$-module. Hence $\mathrm{M}$ is tsemisimple by Proposition $(3.4)(1 \Rightarrow 3)$, also $\mathrm{M}$ is injective by (Proposition (3.4) $((1) \Rightarrow(5))$. It follows that $\mathrm{M}$ is strongly t-semisimple by (Corollary (2.15))

$(2) \Rightarrow(1)$ By condition (2) every nonsingular module M is strongly t-semisimple, hence every nonsingular module $\mathrm{M}$ is t-semisimple. Thus every nonsingular is semisimple by (Remark and Examples 2.2(6)). It follows that $\mathrm{R}$ is t-semisimple by (Proposition (3.4) (4) $\Rightarrow(1))$.

$(1) \Rightarrow(3)$ By (Proposition (3.4) (1) $\Rightarrow(6)), M=Z_{2}(M) \oplus M^{\prime}$ for some injective R-module $M^{\prime}$ by But $M^{\prime} \cong \frac{M}{Z_{2}(M)}$ which is nonsingular module. Hence $M^{\prime}$ is t-semisimple by (proposition $\left.(3.4)(1) \Rightarrow(4)\right)$. Thus $M^{\prime}$ is t-semisimple and injective, so $M^{\prime}$ is strongly t-semisimple by Corollary (2.15).

(3) $\Rightarrow(1) M=Z_{2}(M) \oplus M^{\prime}$, where $M^{\prime}$ is strongly t-semisimple. Hence $M^{\prime}$ is t-semisimple. But $M^{\prime} \cong \frac{M}{Z_{2}(M)}$ which is nonsingular, so $M^{\prime}$ is nonsingular t-semisimple. Thus $M^{\prime}$ is semisimple by Remarks and Examples 2.2(6). But $M^{\prime}$ is injective. Thus $R$ is t-semisimple by (Proposition $(3.4)(6) \Rightarrow(1)$ ).

\section{References}

[1] Abas, M.S.(1991). On Fully Stable Modules, Ph.D. Thesis, College of Science, University of Baghdad.

[2] Asgari, Sh., Haghany, A. (2011).t-Extending modules and t-Baer modules, Comm.Algebra, 39:1605-1623.

[3] Asgari, Sh., Haghany, A.,Tolooei Y. (2013). T-semisimple modules and T-semisimple rings comm. Algebra,41:5,1882-1902. 
[4] Asgri, Sh., Haghany, A.(2010).Densely co-Hopfian modules. Journal of Algebra and Its Aplications 9:989-1000.

[5] Chatters, A. W., Khuri, S. M. (1980). Endomorphism rings of modules over nonsingular CS rings, J. London Math. Soc. 21:434-444 .

[6] Chen, J., Ding, N., Yousif, M. F. (2004). On Noetherian rings with essential socle, J.

[7] Clark, J., Lomp, C., Vanaja N., Wisbauer, R. (2006). Lifting Modules. Frontiers inMathematics, Birkhauser Verlag, Basel.

[8] Dung, N. V., Huynh, D. V., Smith, P. F, Wisbauer, R. (1994). Extending Modules.Pitman Research Notes in Mathematics 313, Longman, Harlow.

[9] Goodearl K.R., Ring Theory, Non Singular Rings and Modules,(1976) Marcel Dekker, Inc. New York and Basel.

[10] Kasch F.Modules and Rings (1982), Acad. Press, London.

[11] Mijbass A .S. , ,1997." Quasi Dedekind Modules ” ,Ph.D .Thesis ,College of Science University of Baghdad .

[12] Lam, T. Y. (1998). Lectures on Modules and Rings. Graduate Texts in Mathematics, Vol. 189, Springer-Verlag, New York/Berlin.

[13] Patrick F. Smith,(2015) .Fully Invariant Multiplication Modules. Palestine Journal of Mathematics, 4: 462470.

[14] Tamadher A. I.(2015). Modules related to Rickart Modules. Ph.D. Thesis, College of Science,Al- Mustansiriyah University.

[15] Wisbauer R. (1991).Foundations of Modules and Rings theory, reading: Gordon and Breach. 
